\title{
Interrupted Resistance Training and BMD in Growing Rats
}

Authors

Affiliations

\author{
J. K. Godfrey ${ }^{1}$, B. D. Kayser ${ }^{2}$, G. V. Gomez ${ }^{1}$, J. Bennett ${ }^{3}$, S. V. Jaque ${ }^{4}$, K. D. Sumida
}

${ }^{1}$ Department of Biological Sciences, Chapman University, Orange, United States ${ }^{2}$ Department of Integrative and Evolutionary Biology, University of Southern California, Los Angeles, United States ${ }^{3}$ College of Educational Studies, Chapman University, Orange, United States

${ }^{4}$ Department of Kinesiology, California State University, Northridge, United States

\author{
Key words \\ - tibia \\ - DXA \\ - osteocalcin \\ - deoxypyridinoline \\ - 3-pt bending test
}

accepted after revision January 26, 2009

Bibliography DOI 10.1055/s-0029-1202823 Published online:

April 20, 2009

Int J Sports Med 2009; 30:

579-584 @ Georg Thieme Verlag KG Stuttgart · New York ISSN 0172-4622

\section{Correspondence}

Dr. K. D. Sumida

Department of Biological Sciences

Chapman University

One University Drive

92866 Orange

United States

Tel.: + 714/997/69 95

Fax: $+714 / 532 / 6048$

sumida@chapman.edu

\section{Abstract \\ $\nabla$}

A resistance training program, where the exercise was uninterrupted (UT, i.e. continuous repetitions) was compared against another resistance training program where the exercise was interrupted (IT, i.e. 2 exercise sessions during a training day) for enhancing bone modeling and bone mineral density (BMD) in maturating animals. The total volume of work performed between the two resistance training programs was equivalent by design. Young male rats $(\sim 8$ weeks old) were randomly divided into Control (Con, $\mathrm{n}=8)$, UT $(\mathrm{n}=8)$ and IT $(\mathrm{n}=7)$ resistance trained groups. The UT and IT groups were conditioned

\section{Introduction}

\section{$\nabla$}

A vast majority of the literature pertaining to exercise and its role in preventing osteoporosis has focused upon the mature adult and elderly. However, it is becoming evident that activity programs during childhood may play a significant role in delaying the onset, or reducing the severity, of osteoporosis later in life [15]. In support, puberty marks a period of enhanced bone deposition [1] and longitudinal studies suggest that physical activity during childhood may be associated with greater bone density in adulthood [21]. High-impact activities (e.g. jumping) or resistance exercises (e.g. strength training) have been advocated as a putative method to stimulate an osteogenic response and promote the elevation in bone mineral density. Nevertheless, our understanding of the most optimal exercise regimen for maximal stimulation of bone accrual during the maturating period is currently unknown.

Studies by Robling et al. $[13,14]$ characterized an exercise protocol with the potential to maximize the bone formation response. Using anesthetized animals, they loaded the bone at discrete inter- to climb a vertical ladder with weights appended to their tail 3 days/week for 6 weeks. After the 6 week training regimen (Mean $\pm S D$ ), tibial BMD (assessed via DXA) was significantly greater for UT $\left(0.237 \pm 0.008 \mathrm{~g} / \mathrm{cm}^{2}\right)$ and IT $\left(0.238 \pm 0.005 \mathrm{~g} / \mathrm{cm}^{2}\right)$ compared to Con $\left(0.223 \pm 0.004 \mathrm{~g} / \mathrm{cm}^{2}\right)$. Further, serum osteocalcin $(\mathrm{OC})$ was significantly greater for UT ( $45.65 \pm 2.83 \mathrm{ng} / \mathrm{ml})$ and IT ( $46.33 \pm 4.60 \mathrm{ng} /$ $\mathrm{ml})$ compared to Con $(37.86 \pm 4.04 \mathrm{ng} / \mathrm{ml})$. There was no significant difference in BMD or serum OC between UT and IT groups. The results indicate that both resistance training programs were equally effective in elevating BMD in growing animals.

vals during a day (separated by several hours) and reported that interrupting an exercise bout was more effective in eliciting an osteogenic response than a single loading session per training day $[13,14]$. From these experiments, Turner [17] interpreted these results suggesting that bone cells become desensitized to prolonged mechanical loading whereas recovery periods can restore the mechanosensitivity thereby augmenting the osteogenic stimulus culminating in an even greater elevation in bone mineral density compared to the single loading exercise session. While these studies $[13,14,17]$ were extremely promising, they were performed on adult rats under ether-induced anesthesia. Thus, the potential benefit of using interrupted bouts of exercise to maximize the osteogenic response in conscious, growing animals remains unresolved.

To date, we are aware of only two studies that have specifically examined the issue of using interrupted exercise programs to maximize bone deposition in conscious animals during the growth period. Umemura et al. [20] was the first to investigate the potential for using intervals between exercise bouts in conscious maturating 
female rats to augment the osteogenic response. They employed a jumping protocol (i.e. high impact exercise) and failed to observe any difference in bone mass from rats exposed to two bouts of exercise, separated by $6 \mathrm{~h}$ of recovery, within a training day compared to a single continuous, uninterrupted bout of exercise during a training day, where the total number of repetitions was equivalent between the jump training programs [20]. Similarly, using a ladder climbing task to mimic resistance exercise, we previously reported equivalent elevations in bone mineral density between interrupted (3 times per training day separated by $4-5 \mathrm{~h}$ of recovery) and continuous resistance training where the volume of work for each resistance trained group was equivalent by design [5]. While speculative, in both the study by Umemura et al. [20] employing high-impact activity as well as our prior study involving resistance training [5], it is possible that more recovery time (i.e. greater than $6 \mathrm{~h}$ ) was necessary to allow the mechanosensors to reset between exercise bouts for the interrupted training protocol. Failure to allow the mechanosensors to reset could account for the equivalent elevations in bone between the continuous vs. interrupted exercised groups.

The purpose of the current study was to determine if a resistance training program, where sessions were separated into two discrete bouts during an exercise training day (separated by 10$12 \mathrm{~h}$ ), was more effective than a continuous bout of resistance training, on markers of bone modeling, bone mineral density (BMD), and bone strength in maturating animals. We hypothesized that interrupted resistance training would be more effective for stimulating an elevation in bone mineral density compared to uninterrupted resistance training in maturating male rats, culminating in added mechanical bone strength. These results could give insight into the optimal resistance training regimen to employ that would maximize peak bone accrual during the growth period and minimize the onset or severity of osteoporosis during old age.

\section{Materials and Methods}

$\nabla$

\section{Animals}

The experimental protocol for this study was pre-approved by the Chapman University Institutional Review Board and in accord with the Public Health Service policy on the use of animals for research. Twenty-four male Sprague Dawley rats (initially $\sim 225$ grams, $\sim 8$ weeks old) obtained from Charles River Laboratories (Wilmington, MA) were housed individually and maintained on a reverse $12 / 12 \mathrm{~h}$ light/dark cycle. Food and water were provided ad libitum throughout the experimental period. The animals were acclimated to their living conditions for 1 week. Then they were randomly separated into a control group (Con, $n=8$ ), a resistance trained group where the animals performed continuous, uninterrupted repetitions on a given training day (UT, $n=8)$, or another resistance trained group where the animals performed repetitions that were interrupted twice during a training day (IT, $\mathrm{n}=8)$.

\section{Resistance training}

The strength training regimen has previously been described by our laboratory where we have demonstrated an osteogenic response $[5,16]$. Briefly, the animals were required to climb a vertical ladder with weights appended to their tail. There were 26 rungs across the $1 \mathrm{~m}$ ladder. The animals were positioned to ensure that they performed each sequential step, where one repetition along the ladder required 26 total lifts by the animal (or 13 lifts per limb). The resistance trained animals were operantly conditioned to climb the ladder in order to avoid a vat of water beneath them. The exercised animals trained 3 days/week for a total of 6 weeks. The control animals were handled on the same days and times as the trained groups in order to minimize any stress attributable to handling. All animals were weighed at the beginning of the week to monitor weight gains and, for the resistance trained animals, to help determine the amount of mass to append to their tails for the remainder of the week. All resistance trained animals started with $30 \%$ body mass (BM) appended to their tail at week one. At week two they were carrying $60 \%$ BM. At week three they were carrying 90\% BM. At week four they were carrying $120 \%$ BM. At week five they were carrying $150 \% \mathrm{BM}$, where they maintained this resistance until the end of week 6 . For the UT group, the animals performed 6 consecutive ladder climbs on a given training day. The 6 ladder climbs constituted the maximum amount of consecutive repetitions that the UT animals could achieve. For the IT group, the animals performed 3 ladder climbs twice during a training day where $10-12 \mathrm{~h}$ separated a bout of exercise. Thus, the total number of ladder climbs (i.e., total repetitions) in a given training day was equivalent between the UT and IT groups. The resistance (i.e., the mass appended to their tail plus their body mass) and the distance covered served to equate the total volume of work between the UT and IT groups throughout the 6 week training period. It should be noted that one of the animals in the IT group refused to climb the ladder during the third week of training. It has been our experience that this can occur on rare occasions and for unknown reasons. This animal was subsequently eliminated from the IT group which accounts for the reduction in sample size (i.e., $\mathrm{n}=7$ ).

\section{Experimental protocol}

To minimize any residual effect of the last training bout, animals were sacrificed $48 \mathrm{~h}$ after their final training session. The Flexor Hallucis Longus (FHL) was rapidly dissected from the right hindlimb, weighed, and immediately frozen in liquid nitrogen for the subsequent determination of protein content. The FHL was chosen as this has previously been reported to elevate in protein content as a result of the ladder climbing exercise $[5,7,16]$. The left hindlimb was rapidly amputated, positioned, and frozen in liquid nitrogen for the assessment of bone mineral density of the tibia and bone mechanical properties. Blood samples were collected, allowed to clot, centrifuged, and the serum was frozen for the subsequent measurement of serum osteocalcin (OC). Since osteocalcin is a protein secreted by osteoblasts, an elevation in this serum marker would suggest more bone formation. Finally, a syringe was used to extract urine directly from the bladder and immediately frozen for the subsequent measurement of deoxypyridinoline (DPD) and creatinine. DPD is a cross-link of Type I collagen found in bone and is released when osteoclasts are active. An elevation in this marker would suggest more bone resorption. Thus, an examination of serum OC and urinary DPD would help to determine the mechanism of bone formation. All tissue, serum, and urine samples were kept at $-80^{\circ} \mathrm{C}$ until their analyses.

\section{Chemical analyses}

Protein concentration in the FHL was assessed [9] as an indirect indicator of training (i.e., muscle hypertrophy). A sandwich 
Table 1 Body Mass.

\begin{tabular}{|c|c|c|}
\hline Group & Initial Body Mass (grams) & Final Body Mass (grams) \\
\hline Con & $307.0 \pm 20.4$ & $495.2 \pm 20.1$ \\
\hline UT & $305.9 \pm 10.3(P=0.899)$ & $459.3 \pm 28.4(P=0.065)$ \\
\hline IT & $307.1 \pm 16.3(P=0.984)$ & $469.4 \pm 55.8(P=0.193)$ \\
\hline \multicolumn{3}{|c|}{$\begin{array}{l}\text { Con = Control Group }(n=8) \text {, UT = Uninterrupted Resistance Trained Group }(n=8) \text {, } \\
\text { and IT = Interrupted Resistance Trained Group }(n=7) \text {. Values are the mean } \pm \text { SD. No } \\
\text { significant difference between groups. P values reflect a comparison to the Control } \\
\text { group }\end{array}$} \\
\hline
\end{tabular}

enzyme-linked immunosorbent assay (ELISA, Biomedical Technologies, Inc., Stoughton, MA) was used to determine serum osteocalcin levels (i.e., an indicator of osteoblast activity). The intra-assay variation was $<3.5 \%$ and the inter-assay variation was $<6 \%$. Urinary deoxypyridinoline (i.e., an indicator of osteoclast activity) was measured using a competitive enzyme immunoassay (EIA, Quidel Corp., San Diego, CA). The intra-assay variation was $<6 \%$ and the inter-assay variation was $<7.5 \%$. Urinary creatinine was measured using an enzyme assay and picric acid as the color reagent (Quidel Corp., San Diego, CA). The intraassay variation was $<2 \%$ and the inter-assay variation was $<4 \%$. A microplate reader (MaxLine, Molecular Devices Corp., Sunnyvale, $C A$ ) was used with the absorbance set at $450 \mathrm{~nm}$ for the ELISA, $405 \mathrm{~nm}$ for the EIA, or $490 \mathrm{~nm}$ for the microassay using picric acid. A standard curve was generated for all chemical analyses and controls were run to ensure quality. For all standard curves, the correlation coefficient (Pearson's Product Moment Correlation for linear standard curves, i.e. Protein and Creatinine, and Coefficient of Determination for non-linear standard curves, i.e. Osteocalcin and Deoxypyridinoline) was greater than 0.97. Finally, a Dual Energy X-ray Absorptiometer (DXA - GE Lunar Prodigy, Chicago, IL) employing the small animal software module (version 6.81) was used to assess the BMD of the left tibia. The left hindlimb was thawed, positioned, and the tibia was scanned. Three consecutive measurements were performed with the hindlimb repositioned between each scan. The reported BMD was the average of three scans and the coefficient of variation for repeated scans (mean \pm standard error) was $0.86 \pm 0.08 \%$.

\section{Biomechanical three- point bending tests}

The mechanical properties of bone were measured using a threepoint bending rig placed onto the stage of a texture analyzer instrument (TA-XT2, Texture Technologies, Ramona, CA). Following the DXA scans, hindlimbs were thawed and all soft tissues were removed from the left tibia. The bone was submerged in saline for $20 \mathrm{~h}$ prior to testing at room temperature. Prior to testing, the instrument was calibrated using a standard weight. Then the tibia was patted dry and secured to the rig. The span of the two support points was $18 \mathrm{~mm}$ and the deformation rate was $0.9 \mathrm{~mm} / \mathrm{sec}$. A medial to lateral force was applied to the midshaft of the bone. The maximal load to failure (Fmax, units = $\mathrm{N}$ ) and energy to failure (EF, determined from the area under the load-deformation curve to the fracture point, units $=\mathrm{N} \times \mathrm{mm}$ ) was assessed using Texture Expert (v. 1.22, Stable Micro Systems Ltd., Surrey, England, UK).

\section{Calculations and statistics}

Work (i.e. training volume) was calculated as the product of the total mass lifted by the animal (body mass plus the amount of mass appended to the tail), the acceleration due to gravity, and



Fig. 1 Total work (in Joules) performed for each training day during the week by the Uninterrupted resistance trained group (UT, n=8) and the Interrupted resistance trained group (IT, $n=7)$. Values are mean \pm SD. No significant difference between groups.

the distance covered. The total training volume (i.e. work) for UT and IT was expressed in Joules. For weekly total training volume, a Student's t-test was used to determine statistical significance. Total protein in the FHL was calculated as the product of protein concentration and muscle mass. Deoxypyridinoline (nmol/L) was corrected for urine concentration (or dilution), by dividing by the creatinine concentration ( $\mathrm{mmol} / \mathrm{L}$ ) and expressed as the adjusted urinary DPD. Except for the training volume (see above), an ANOVA was employed for all other comparisons, and when a significant $\mathrm{F}$ ratio was identified, a Fisher's PLSD post hoc test was used. The level of significance set was at $\mathrm{P}<0.05$ for all statistical comparisons and the results were expressed as the mean \pm standard deviation (SD).

\section{Results}

$\nabla$

The initial body mass was not significantly different between groups ( $\bullet$ Table 1). After the 6 week resistance training program, the final body mass was similarly not significantly different between groups ( $\bullet$ Table 1). The total training volume for the resistance trained animals was not significantly different between UT vs. IT at any time during the 6 week exercise program ( Fig. 1). The FHL mass and total protein content in the FHL was significantly greater for UT and IT groups when compared to the Con group ( $\bullet$ Table 2 ). There was no significant difference in FHL mass or total protein in the FHL between UT and IT groups.

The bone mineral density from the left tibia from both the UT and IT groups were significantly greater than the Con group (॰ Fig. 2). However, the BMD was not significantly different between UT and IT groups. Serum osteocalcin was significantly greater for both the UT and IT groups compared to controls ( $\bullet$ Fig. 3), but was not significantly different between UT and IT groups. In contrast, the adjusted urinary deoxypyridinoline did not significantly differ between the Con $(119.76 \pm 51.21)$, UT (115.12 \pm 33.96$)$, and IT $(113.29 \pm 30.30)$ groups.

The mechanical properties of the left tibia were significantly elevated for both UT and IT compared to controls as measured from the three point bending test. The maximum force to failure was significantly greater for UT (i.e. $29 \%$ ) and IT (i.e. $25 \%$ ) compared to controls ( $\bullet$ Fig. 4). Similarly, the energy to failure was 
Table 2 Resistance Training Effect on FHL.

$\begin{array}{lll}\text { Group } & \text { FHL Mass (grams) } & \begin{array}{l}\text { FHL Protein } \\ \text { (mg protein/muscle) }\end{array} \\ \text { Con } & 0.337 \pm 0.072 & 68.55 \pm 15.04 \\ \text { UT } & 0.414 \pm 0.048^{*}(P=0.048) & 87.15 \pm 14.22^{*}(P=0.031) \\ \text { IT } & 0.434 \pm 0.086^{*}(P=0.024) & 90.08 \pm 18.86^{*}(P=0.017)\end{array}$

Con $=$ Control Group $(n=8)$, UT = Uninterrupted Resistance Trained Group $(n=8)$, and IT = Interrupted Resistance Trained Group $(\mathrm{n}=7)$. FHL = flexor hallucis longus. Values are the mean \pm SD. *Significant difference vs. Con. There was no significant difference between UT vs. IT

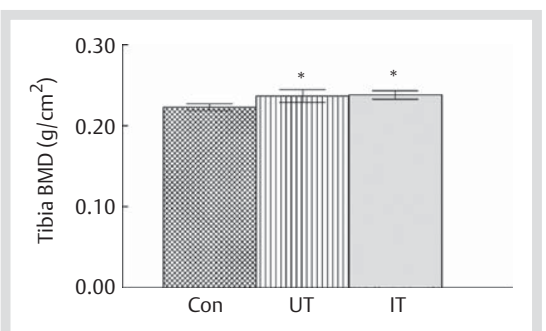

Fig. 2 Bone Mineral Density (BMD) for the left tibia from Controls (Con, $\mathrm{n}=8$ ), Uninterrupted resistance trained group (UT, $n=8)$, and Interrupted resistance trained group $(I T, n=7)$. Values are mean $\pm S D$. *Significant difference vs. Con. $\mathrm{P}<0.0001$ for both UT and IT vs. Con.



Fig. 3 Serum osteocalcin (OC) concentrations from Controls (Con, $n=8)$, Uninterrupted resistance trained group (UT, $n=8)$, and Interrupted resistance trained group $(I T, n=7)$. Values are mean \pm SD. ${ }^{*}$ Significant difference vs. Con. $\mathrm{P}<0.0006$ for both UT and IT vs. Con.

significantly greater for UT (i.e. 60\%) and IT (i.e. 57\%) compared to controls ( $\odot$ Fig. 4). However, the maximal force and energy to failure was not significantly different between UT and IT groups (० Fig. 4).

\section{Discussion \\ $\nabla$}

The uninterrupted and interrupted 6 week resistance training regimens (where the total volume of work was kept constant between UT vs. IT groups) were equally effective for stimulating an increase in tibial BMD in maturating male rats. The osteogenic response for both UT and IT was attributable to an elevation in osteoblast activity, as indicated by an increase in serum OC compared to controls, and not a lower osteoclast activity, as indicated by equivalent levels of adjusted urinary DPD between groups. Both UT and IT groups demonstrated an augmented bone strength as assessed from three point bending tests when compared to controls. While the BMD, bone strength, and serum OC were elevated in both UT and IT compared to controls, there

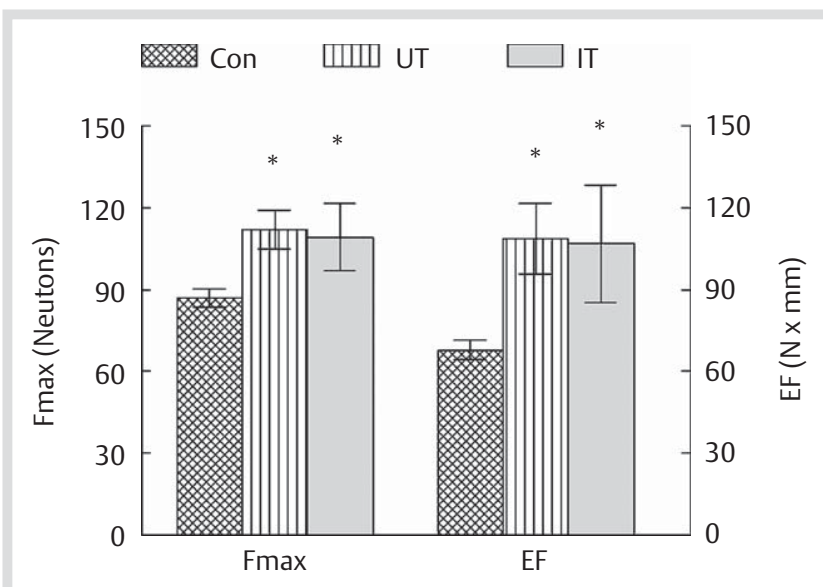

Fig. 4 Bone strength characteristics of the left tibia from Controls (Con, $\mathrm{n}=8$ ), Uninterrupted resistance trained group (UT, $\mathrm{n}=8$ ), and Interrupted resistance trained group $(\mathrm{IT}, \mathrm{n}=7)$. Fmax = maximal force to failure expressed in Newtons. EF = energy to failure (area under the load deformation curve) expressed in Newtons $\times$ millimeters $(\mathrm{N} \times \mathrm{mm})$. Values are mean \pm SD. *Significant difference vs. Con. $\mathrm{P}<0.05$ for both UT and IT vs. Con.

was no significant difference between UT and IT groups. Thus, our results did not support our hypothesis of a greater osteogenic response from IT compared to UT.

Our results on the strength training-induced elevation in BMD are consistent with prior studies that have similarly reported on the effectiveness of exercise in promoting an osteogenic response in maturating animals $[6,8,11,12]$. Further, the current report confirms previous findings in humans $[3,4,10]$ and animals $[16,22,23]$ pertaining to the exercise-induced elevation in osteoblast activity as supported by the significant increase in serum OC for both UT and IT compared to controls. Last, our findings regarding the elevation in bone mechanical properties as a result of resistance training are also consistent with other reports in rats that examined the femur after jumping exercise [11] and tower climbing [12], confirming that relatively small increases in BMD can culminate in large elevations in bone strength.

While our results pertaining to strength training upon the bone are not unique, we note that the equivalent effectiveness from both UT and IT exercise programs is in contrast to the prevailing hypothesis submitted by Turner and Robling [19]. Specifically, the recovery period of $10-12 \mathrm{~h}$ between exercise bouts that we implemented for the IT group should have restored the mechanosensitivity of the bone cells thereby providing a greater osteogenic stimulus for bone formation. We have no explanation for the disparate results between our report and that of Robling et al. $[13,14]$, but we acknowledge several differences between the studies. First, we used conscious animals and a resistance training program that incorporated bouts of exercise 3 days per week. In contrast, Robling et al. $[13,14]$ used anesthetized animals and a daily loading protocol. Next, it is conceivable that the age of the animal might be a factor. In the current study, we used maturating animals whereas adult rats were employed by Robling et al. $[13,14]$. Thus, there is the potential that the hormonal milieu during the growth period, in combination with the exercise, reached a maximal stimulation threshold where the osteocytes became desensitized. In this regard, the first exercise bout for the IT group was sufficient to generate the requisite fluid flow for bone formation [18]. Alternatively, it is possible that even more time than the $10-12 \mathrm{~h}$ we implemented is 
required to restore the bone mechanosensitivity, especially in maturating rats. Collectively, some or all these factors may have contributed to the contrasting results between our study and that of Robling et al. [13,14].

Our findings, however, support the two current studies that specifically examined the potential for implementing interrupted exercise bouts as a method to more effectively augment BMD than continuous exercise in maturating, conscious animals. Umemura et al. [20] used high impact (i.e. jumping) exercise 5 days/week for 8 weeks. They reported no difference in elevating bone mass using a $6 \mathrm{~h}$ recovery between two daily exercise sessions $(2 \times 10$ jumps $)$ compared to a continuous exercise bout $(1 \times 20$ jumps $)$ in maturating rats [20]. Similarly, in a prior resistance training study employing the ladder climbing task, we observed equal effectiveness in elevating BMD and bone strength between continuous versus interrupted resistance training (i.e. 3 bouts per training day separated by $4-5 \mathrm{~h}$ ) after 6 weeks of exercise 3 days/week in growing rats. Taken together, these two prior reports and the results of the current study do not lend support to the contention, at least in conscious maturating animals, that separating a bout of exercise into discrete sessions during a training day is more effective than a single bout of exercise performed on a given training day.

When assessing the physiological benefits of exercise, the use of an animal model helps to minimize many of the confounding variables associated with human cross-sectional studies that are subject to various factors such as: genetics, dietary intake, and activity levels (to name a few). Additional challenges, especially when studying children, include matching the growth velocity between the exercise and control groups [2]. In this regard, the use of maturating rats can minimize many of these confounding variables, but we acknowledge several limitations with use of this animal model. First, we recognize that the epiphyseal plates in rats do not close. As such, we chose to examine the impact of exercise specifically during the growth period in rats that could apply to maturating humans, although this should be done with caution. Second, we measured BMD and markers for bone formation/resorption at a single time point (i.e. the end of the 6 weeks) whereas a better experimental design would have been to measure BMD and markers for bone formation/resorption initially and then throughout the 6 week period to help differentiate the impact of growth in addition to the exercise stimulus. Next, there is always the concern that an observation in animals may not occur in humans. However, the numerous observations in rats have been consistent with the reports in humans pertaining to the impact of exercise on bone. Thus, our experimental design offered a potential insight into the type of resistance training program (i.e. continuous vs. interrupted exercise) that could optimize bone accrual during growth, albeit our results suggest that both resistance training programs were equally effective.

Finally, based upon our observations of the exercised animals in the current study as well our prior report [5], we offer a consideration that could be relevant to humans. The number of repetitions performed by the IT group was predicated on the number of repetitions achieved by the UT group in order to equate the volume of work between the groups per training day. Separating the exercise into two bouts for the IT group was sufficient where animals readily achieved the required work for each exercise session during a training day whereas the UT group struggled to accomplish the last couple of full ladder climbs during an exercise session. In this regard it is possible that even more recovery time is necessary for the mechanosensors to reset. Alternatively, if the mechanosensors were still desensitized for the IT group following the first bout of exercise, then the second bout of exercise would contribute little to the observed increase in BMD. Thus, it is possible that completing only half the work during a training day (i.e. only 3 ladder climbs for the IT group) would provide the requisite stimulus for bone formation. To the extent that this alternative speculation can be extrapolated to an application for humans, exercise that takes the skeletal muscle to exhaustion may not be necessary to stimulate bone accrual during growth. Exercise that does not require taking the skeletal muscle to fatigue would minimize the potential discomfort during the exercise as well as lower the potential for muscle soreness following the activity. Limiting the pain and discomfort associated with exercise could reduce the attrition rate for children and encourage a lifestyle that promotes a healthy skeletal system that could eventually lower the impact or severity of osteoporosis in the later stages of life. Therefore, we acknowledge that further investigations are warranted in growing animals to determine the minimum exercise threshold (e.g. frequency, intensity, etc.), where muscles are not taken to the point of fatigue, that can elicit an osteogenic response equally effective to resistance training where muscles are exercised to exhaustion.

In summary, using conscious animals and a mode of exercise that mimics resistance training, where the volume of work was matched between UT and IT programs, we offer evidence that both exercise regimens were equally effective for stimulating an osteogenic response in maturating male animals. This is supported by an increase in BMD attributable to an augmentation in osteoblast activity as assessed by elevations in serum OC levels for the resistance trained groups compared to controls. In addition, the bone mechanical properties, as measured from three point bending tests, were elevated in both resistance trained groups compared to controls. We conclude that under the conditions of the current study, interrupted resistance training did not provide a greater stimulus for osteogenesis when compared to uninterrupted resistance training during the growth period in rats. We also acknowledge that further investigations are warranted to determine if daily resistance training, where muscles are not overworked, can similarly elicit an osteogenic response equally effective to resistance training every other day where muscles are exercised to exhaustion.

\section{References}

1 Bonjour JPH, Theintz G, Buchs B, Slosman D, Rizzoli R. Critical years and stages of puberty for spinal and femoral bone mass accumulation during adolescence. J Clin Endocrinol Metab 1991; 73: 555-563

2 Bradney M, Pearce G, Naughton G, Sullivan C, Bass S, Beck T, Carlson J, Seeman E. Moderate exercise during growth in prepubertal boys: changes in bone mass, size, volumetric density, and bone strength: a controlled prospective study. J Bone Min Res 1998; 13: 1814-1821

3 Danz AM, Zittermann A, Schiedermaier U, Klein K, Hotzel D, Schonau E. The effect of a specific strength-development exercise on bone mineral density in perimenopausal and postmenopausal women. J Women Health 1998; 7: 701-709

4 Fujimura R, Ashizawa N, Watanabe M, Mukai N, Amagai H, Fukubayashi $T$, Hayashi K, Tokuyama K, Suzuki M. Effect of resistance exercise training on bone formation and resorption in young male subjects assessed by biomarkers of bone metabolism. J Bone Min Res 1997; 12: 656-662

5 Goettsch BM, Smith MZ, O'Brien JA, Gomez GV, Jaque SV, Sumida KD. Interrupted vs. uninterrupted training on BMD during growth. Int J Sports Med 2008; 29: 980-986

6 Honda A, Naota S, Nagasawa S, Shimizu T, Umemura Y. High-impact exercise strengthens bone in osteogenic ovariectomized rats with the same outcome as Sham rats. J Appl Physiol 2003; 95: 1032-1037 
7 Hornberger TA, Farrar RP. Physiological hypertrophy of the FHL muscle following 8 weeks progressive resistance exercise in the rat. Can J Appl Physiol 2004; 29: 16-31

8 Huang TH, Lin SC, Chang FL, Hsieh SS, Liu SH, Yang RS. Effects of different exercise modes on mineralization, structure, and biochemical properties in growing bone. J Appl Physiol 2003; 95: 300-307

9 Lowry $\mathrm{OH}$, Rosebrough NJ, Farr AL, Randall RJ. Protein measurement with the Folin phenol reagent. J Biol Chem 1951; 193: 265-275

10 Menkes A, Mazel S, Redmond RA, Koffler K, Libanati CR, Gundberg CM, Zizic TM, Hagberg JM, Pratley RE, Hurley BF. Strength training increases regional bone mineral density and bone remodeling in middle-aged and older men. J Appl Physiol 1993; 74: 2478-2484

11 Notomi T, Lee SJ, Okimoto N, Okazaki Y, Takamoto T, Nakamura T, Suzuki $M$. Effects of resistance exercise training on mass, strength, and turnover of bone in growing rats. Eur J Appl Physiol 2000; 82: 268-274

12 Notomi T, Okimoto N, Okazaki Y, Tanaka Y, Nakamura T, Suzuki M. Effects of tower climbing exercise on bone mass, strength, and turnover in growing rats. J Bone Min Res 2001; 16: 166-174

13 Robling AG, Burr DB, Turner $\mathrm{CH}$. Recovery periods restore mechanosensitivity to dynamically loaded bone. J Exp Biol 2001; 204: 33893399

14 Robling AG, Hinant FM, Burr DB, Turner CH. Improved bone structure and strength after long-term mechanical loading is greatest if loading is separated into short bouts. J Bone Min Res 2002; 17: 1545-1554
15 Seeman E. An exercise in geometry. J Bone Miner Res 2002; 17: $373-$ 380

16 Smith MZ, Goettsch BM, O'Brien JA, Van Ramshorst RD, Jaque SV, Sumida $K D$. Resistance training and bone mineral density during growth. Int J Sports Med 2008; 29: 316-321

17 Turner $\mathrm{CH}$. Three rules for bone adaptation to mechanical stimuli. Bone 1998; 23: 399-407

18 Turner $\mathrm{CH}$, Ichiro O, Takano Y. Mechanotransduction in bone: role of strain rate. Am J Physiol 1995; 269: E438-E442

19 Turner $\mathrm{CH}$, Robling AG. Designing exercise regimens to increase bone strength. Exerc Sport Sci Rev 2003; 31: 45-50

20 Umemura $Y$, Sogo $N$, Honda A. Effects of intervals between jumps or bouts on osteogenic response to loading. J Appl Physiol 2002; 93: 1345-1348

21 Welten DC, Kemper HCG, Post BG, Van Mechelen W, Twisk J, Lips P, Teule $G J$. Weight-bearing activity during youth is a more important factor for peak bone mass than calcium intake. J Bone Miner Res 1994; 9: 1089-1096

22 Westerlind KC, Fluckey JD, Gordon SE, Kraemer WM, Farrell PA, Turner $R T$. Effect of resistance exercise training on cortical and cancellous bone in mature male rats. J Appl Physiol 1998; 84: 459-464

23 Yeh JK, Chung CL, Aloia JF. Effects of exercise and immobilization on bone formation and resportion in young rats. Am J Physiol 1993; 264: E182-E189 